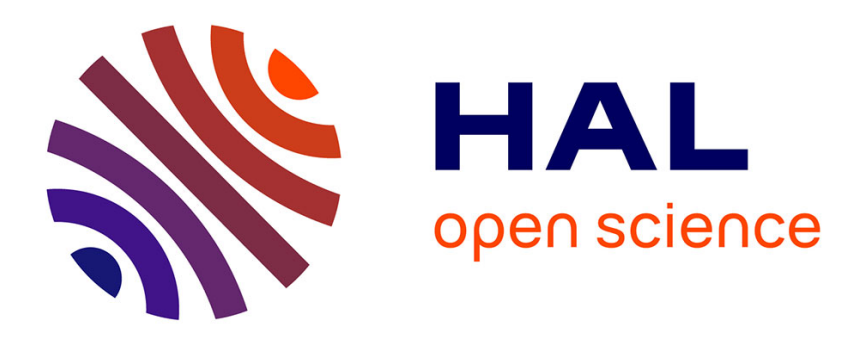

\title{
Quand le merveilleux saisit nos sens: spectaculaire et féeries en France (XVIIe-XIXe siècle)
}

Roxane Martin

\section{To cite this version:}

Roxane Martin. Quand le merveilleux saisit nos sens: spectaculaire et féeries en France (XVIIe-XIXe siècle). Sociétés \& Représentations, 2011, 31, pp.17-33. 10.3917/sr.031.0017 . hal-02482814

\section{HAL Id: hal-02482814 \\ https://hal.univ-lorraine.fr/hal-02482814}

Submitted on 18 Feb 2020

HAL is a multi-disciplinary open access archive for the deposit and dissemination of scientific research documents, whether they are published or not. The documents may come from teaching and research institutions in France or abroad, or from public or private research centers.
L'archive ouverte pluridisciplinaire HAL, est destinée au dépôt et à la diffusion de documents scientifiques de niveau recherche, publiés ou non, émanant des établissements d'enseignement et de recherche français ou étrangers, des laboratoires publics ou privés. 
Cet article est paru dans: Sociétés \& représentions, $\mathrm{n}^{\circ} 31$ : «Le Spectaculaire à l'œuvre », numéro coordonné par Pascale Goetschel, Publications de la Sorbonne, avril 2011, p. 19-33.

\section{Quand le merveilleux saisit nos sens : spectaculaire et féeries en France (XVII ${ }^{\mathrm{e}}-\mathrm{XIX}^{\mathrm{e}}$ siècles)}

S'il demeure une forme dans l'histoire théâtrale française qui favorise tout particulièrement l'analyse du spectaculaire, entendu à la fois comme forme d'émerveillement et appel aux sens, c'est incontestablement la féerie. Théâtre de l'imaginaire pur, elle n'est pas assujettie aux lois du vraisemblable et peut tout naturellement justifier l'usage d'une machinerie théâtrale poussée jusqu'aux limites de ses possibilités, renouvelant chaque fois, selon les époques, les défis du spectaculaire et de l'illusionnisme total. Les "grandes féeries » du Théâtre du Châtelet, dans les dernières décennies du XIX ${ }^{\mathrm{e}}$ siècle, se donnent comme de parfaits exemples. Mobilisant un personnel technique et dramatique nombreux, elles offrent à un public émerveillé des transformations scéniques surprenantes : des forêts qui marchent, des objets qui s'animent, des membres qui se détachent des corps, des animaux et des hommes qui grossissent à vue, des mots qui, prononcés sur scène, font surgir les éléments qu'ils évoquent ${ }^{1}$. La « grande féerie» de la deuxième moitié du XIX ${ }^{\mathrm{e}}$ siècle place le spectacle au cœur même de son écriture. Théâtre de la matière, elle met en mouvement les corps et les objets, explore la plasticité du langage et sa capacité à signifier le réel, soumet les techniques de l'illusion à sa recherche de l'effet. Aussi n'est-il pas surprenant de voir intégré, un an à

1. Les exemples sont nombreux; mentionnons simplement le cas de Turlututu, dans la pièce éponyme, qui se voit transformé en arrosoir aussitôt après avoir prononcé le mot (voir Clairville, Monnier, Martin, Turlututu, chapeau pointu, grande féerie en 3 actes et 30 tableaux, Paris, Lévy frères, [1858], p. 13). 
peine après son invention, le cinématographe au sein des spectacles féeriques du Châtelet. Le 14 novembre 1896 est reprise sur ce théâtre La Biche an bois ${ }^{1}$ qui offre à Jacques Ducom, le projectionniste des frères Lumière, l'occasion de réaliser pour Gaumont l'un des premiers films de fiction de l'histoire, inaugurant un genre cinématographique dont Georges Méliès saura ensuite se faire une spécialité ${ }^{2}$. Tourmenté par des démangeaisons nasales provoquées par une méchante fée, le héros de la pièce voit son nez prendre tout à coup des proportions gigantesques. Sur le mur du fond est projeté le nez de l'acteur, que l'on voit grossir, rougir et s'allonger démesurément, puis éclater ; une foule de lutins sort du nez, exécute une danse infernale en frappant l'appendice nasal à l'aide de piques et de marteaux ${ }^{3}$. Inutile de souligner l'effroi, la surprise ou tout simplement l'enthousiasme qu'ont pu ressentir ce soir-là les spectateurs du Châtelet. En utilisant les moyens matériels du spectacle pour produire un effet d'émerveillement, la féerie remplit le sens de ce que l'on nomme communément «le spectaculaire ».

\section{LE(S) SENS DU SPECTACULAIRE}

Pourtant le mot est anachronique pour caractériser la féerie théâtrale de la deuxième moitié du XIX ${ }^{\mathrm{e}}$ siècle. Depuis les travaux de Philippe Roger, on sait que l'adjectif «spectaculaire» apparait

1. La Biche au bois on le Royaume des fées, vaudeville-féerie en 4 actes et 16 tableaux d'Ernest Blum, Hippolyte \& Théodore Cogniard et Raoul Toché, fut créée au théâtre de la Porte-Saint-Martin le 29 mars 1845. Elle fut remaniée en " grande féerie en 4 actes et 30 tableaux » lors de sa reprise en 1896 au théâtre du Châtelet, devenu, après la destruction du Boulevard du Temple en 1862, le temple de la féerie à grand spectacle.

2. Georges Méliès participe d'ailleurs lui-même à la confection de « clous » cinématographiques projetés dans les féeries du Châtelet qu'il réintègre ensuite dans ses propres films. Pour Les Quatre Cents Coups du diable (Châtelet, 1905), il crée l'épisode de la «voiture céleste » qu'il a combiné ensuite avec des séquences de la féerie filmées à l'issue des représentations. Ce film est aujourd'hui consultable sous le titre Les Quatre Cents Farces du diable (1906, rééd. Lobster, DVD, 2009).

3. Voir Jacques Ducom, Le Cinématographe muet, sonore, parlant, Paris, Albin Michel, 1911. Sur les premiers lieux et productions du cinématographe, voir également: Jean-Jacques Meusy, Paris-Palaces ou le Temps des cinémas (18941918), Paris, CNRS éd., 2002. Pour réaliser cet effet, Ducom avait superposé des images fixes coloriées (celles figurant le nez dans ses différentes tailles) à la projection cinématographique de la danse des lutins. 
en $1908^{1}$ et remplace le mot «spectaculeux » qui, selon Pierre Larousse, est synonyme de "pompeux » et désigne la chose qui « ressemble à un spectacle $»^{2}$. Couramment employé par la critique pour qualifier, entre autres, les productions féeriques du Châtelet, l'adjectif renvoie à " un trop-plein de spectacle : un excès »; il est "le signe ostentatoire du spectacle en tant que machine, pompe, appareil $»^{3}$. Le terme cède ensuite la place à l'adjectif « spectaculaire » qui désigne d'abord tout ce qui a trait au spectacle pour ensuite prendre les acceptions définies comme telles par le Trésor de la langue française:

SPECTACULAIRE : Qui frappe la vue, l'imagination par son caractère remarquable, les émotions, les réflexions suscitées. [...] Qui concerne les spectacles, les représentations théâtrales, musicales, chorégraphiques. [...] Qui produit, qui cherche à produire un effet visuel, émotionnel. [...] Qui est assimilé à un spectacle par la performance, le sens artistique présenté, les émotions provoquées. ${ }^{4}$

Si le mot "spectaculeux» désignait un usage excessif de la machinerie théâtrale, le concept de spectaculaire englobe les conditions matérielles du spectacle et leur impact sur le public. Autant dire que son analyse ne peut faire l'économie d'une étude de la réception des œuvres; elle consiste à mesurer la façon dont les éléments scéniques sont utilisés pour produire l'émotion.

On peut alors s'étonner de voir apparaittre si tardivement l'adjectif «spectaculaire» dans la langue française. Comme le précise Isabelle Moindrot :

L'introduction d'un mot dans la langue ne traduit pas forcément la naissance subite d'une réalité jusqu'alors inexistante. Mais elle révèle en tout cas une prise de conscience de ce que cette réalité recouvre de singulier, d'original, nécessitant la production d'un terme neuf pour le reconnaitre. Ainsi, le mot «spectaculaire» est-il apparu non pas en même temps que des pratiques de scène particulièrement

1. Voir Philippe Roger, "Spectaculaire, Histoire d'un mot », in Christine Hamon-Siréjols et André Gardies (dir.), Le Spectaculaire, Lyon, Cahiers du GRITEC / Aléas, 1997.

2. Pierre Larousse, Grand dictionnaire universel du XIX ${ }^{e}$ siècle, Paris, 18661877, entrée « spectaculeux ».

3. Philippe Roger, loc. cit., p. 9-10.

4. Trésor de la langue française informatisé, ATILF, CNRS éd., 2004, entrée « spectaculaire ». 
sensationnelles, ni même dans l'ardeur des débats autour des excès du réalisme romantique, mais au moment même où l'art de la mise en scène est devenu une réalité admise comme telle, et débattue sur la place publique. ${ }^{1}$

L'apparition du mot «spectaculaire » est concomitante, en effet, aux premières théorisations de la mise en scène. Ce facteur est essentiel pour comprendre les enjeux esthétiques, politiques et sociaux qui sous-tendent la réception du «spectaculaire » et qui conduisent la critique tantôt à promouvoir le théâtre «à grand spectacle », tantôt à le condamner. Tandis que les auteurs de la première moitié du XIX ${ }^{\mathrm{e}}$ siècle avaient élaboré des dramaturgies intégrant les éléments scéniques dans le but de produire l'émotion, les réformateurs du théâtre, au début du $\mathrm{XX}^{\mathrm{e}}$ siècle, se posent la question du sens que la mise en scène peut accorder à l'œuvre dramatique. Deux conceptions du spectacle ici s'opposent. Les dramaturges romantiques, prolongeant les ambitions des philosophes des Lumières qui souhaitaient accorder au théâtre un rôle dans l'éducation du peuple, avaient combiné, au sein même de leur dramaturgie, le verbe, le geste, le décor, la musique de façon à toucher le public et, par ce biais, à permettre au drame de remplir sa fonction esthétique, sociale et politique: "Jamais dans ses travaux, ['auteur] ne perd un seul instant de vue le peuple que le théâtre civilise » écrivait Victor Hugo dans la préface de Marie Tudor (1833) ${ }^{2}$. Et l'acte civilisateur du théâtre s'accomplit précisément par le biais de l'émotion. Autrement dit, l'effet produit par le spectacle est au cœur même des enjeux de la composition dramatique puisque c'est par son biais que le théâtre accomplit sa

1. Isabelle Moindrot, "Introduction », in Isabelle Moindrot (dir.), Le Spectaculaire dans les arts de la scène du Romantisme à la Belle Époque, Paris, CNRS éd., 2006, p. 10.

2. Cette foi en la vocation « civilisatrice» du drame n'est pas propre à Hugo; on la retrouve formulée, entre autres, par les mélodramaturges du début du XIX ${ }^{\mathrm{e}}$ siècle et, de façon plus générale, par les auteurs libéraux. Ainsi, François Guizot dans son «Étude sur Shakespeare », écrit : « il faut que [le poète dramatique] élève et civilise, pour ainsi dire, la foule qu'il appelle à ses fêtes : comment agir sur les hommes assemblés, sinon en s'adressant à ce qu'il y a de plus général et de plus élevé dans leur nature ? C’est seulement en sortant de la vie et des intérêts individuels que l'imagination s'exalte, que l'âme s'agrandit, que les plaisirs deviennent désintéressés et les affections généreuses, que les hommes peuvent se rencontrer dans ces émotions communes dont les transports font retentir le théâtre » (in CEuvres complètes de Shakespeare, Paris, Didier \& C Cie, 1860, p. 3-4). 
mission sociale ${ }^{1}$. Au tournant des $\mathrm{XIX}^{\mathrm{e}}$ et $\mathrm{XX}^{\mathrm{e}}$ siècles, la fonction des éléments du spectacle se pense différemment. Les directeurs de théâtre sous le Second Empire avaient considérablement développé les moyens scéniques alloués à la mise en scène ${ }^{2}$. La loi de 1864, en proclamant la liberté industrielle des spectacles, et les travaux haussmanniens de rénovation urbaine, en redessinant la géographie des théâtres parisiens et, dans le même coup, la répartition sociale des publics en fonction des salles, avaient changé les donnes de la production théâtrale. Davantage soumis à la concurrence et désireux de séduire un public moins composite et avide de sensations fortes, les grands théâtres de la capitale avaient cédé au prestige de la mise en scène, quitte parfois, à force de surenchère sur les effets, à conduire l'espace scénique au bord

1. On pourrait multiplier les exemples pour illustrer ce point car c'est finalement le théâtre du XIX ${ }^{\mathrm{e}}$ siècle dans son ensemble qui, avec le dénominateur commun du spectaculaire, a remodelé les codes traditionnels de l'écriture théâtrale afin de mettre en œuvre une esthétique de l'illusion et de la fascination. Citons simplement le mélodrame qui, l'un des premiers, a soumis les éléments du spectacle à sa mission civilisatrice. Les valeurs morales et la conception du monde qu'il défend au début du XIX ${ }^{\mathrm{e}}$ siècle sont en effet inculquées à son public par le biais d'une combinaison savamment réfléchie entre discours, gestuelle, picturalité scénique et langage musical. Ainsi, c'est bien parce que la victime innocente use d'un vocabulaire propre à sa condition, prend des attitudes qui renvoient à une typologie gestuelle codifiée - «la douleur placera une main sur son front» ironisaient à ce propos les auteurs du Traité $d u$ mélodrame (Paris, Delaunay, 1817, p. 47) -, laisse le soin à l'orchestre de peindre son désespoir par des phrases musicales qui renvoient elles-mêmes à des codes bien connus du public - «Un cantabile annonçait une princesse infortunée ; un agitato un amant désespéré [...] ; la flûte s'emparait de la mélancolie, le basson et l'alto exprimaient le remords » écrivaient les rédacteurs du Ménestrel ( $\mathrm{n}^{\circ}$ du 19 janvier 1834) -, livre son désespoir dans des décors sombres qui renforcent la misère de sa condition, qu'elle peut toucher le public et, par ce biais, le convaincre de sa vertu. L'émotion ressentie devant le spectacle de l'injuste déshonneur est fondamentale au spectacle mélodramatique. C'est par son biais que le genre parvient à bâtir un partage net entre vices et vertus et à convaincre le public qu'il existe une juste providence sachant condamner les méchants et récompenser les bons. Les éléments scéniques participent de sa rhétorique.

2. Notons que l'expression «mise en scène » apparâtt dans la langue française en même temps que les dramaturgies qui intègrent les éléments du spectacle dans le but de produire l'émotion et de défendre, par son biais, des valeurs civiques et/ou religieuses. Les auteurs de féeries et de mélodrames, au tout début du XIX ${ }^{\mathrm{e}}$ siècle, sont les premiers à se revendiquer «metteurs en scène » (sur ce point, nous renvoyons à notre étude «L'apparition des termes "mise en scène" et "metteur en scène" dans le vocabulaire dramatique français ", dans Mara Fazio et Pierre Frantz (dir.), La Fabrique du théâtre. Avant la mise en scène (1650-1880), Paris, Desjonquères, 2010, p. 19-31). 
de la saturation. La question du pouvoir de la scène s'inscrivit alors au cœur des débats. Et ces débats se nouèrent autour de la mise en scène des «chefs-d'œuvre» du répertoire ${ }^{1}$. S'occupant plus précisément de définir la fonction des éléments scéniques dans le théâtre "littéraire », les réformateurs du théâtre conférèrent à la mise en scène un rôle d'interprétation: "Par mise en scène nous entendons : le dessin d'une action dramatique » précisait Jacques Copeau, n'omettant pas d'opposer cette nouvelle façon de concevoir le travail scénique aux autres pratiques de l'époque :

À cette mise en scène-là, qui concerne l'interprétation, nous ne saurions apporter trop d'étude. À l'autre, qui a trait aux décors et aux accessoires, nous ne voulons pas accorder d'importance. ${ }^{2}$

On comprend alors toute l'utilité de l'adjectif «spectaculaire » qui vint, dans le discours des promoteurs du "théâtre d'art", caractériser le théâtre à sensation qui, selon eux, était entièrement voué à une recherche de l'émotion facile et multipliait les "effets » dans un but simplement commercial. Aussi Copeau pouvait-il ajouter :

Il est à craindre que de tels procédés en s'ajoutant au drame, qu'une telle et si constante recherche de l'effet - toujours défaillante, d'ailleurs, si elle s'applique aux grands ouvrages classiques - ne favorisent progressivement une production dramatique toute artificielle, grossière et presque barbare. ${ }^{3}$

La mise en scène, nouvellement définie, rejetait donc les procédés $\mathrm{du}$ théâtre «à grand spectacle». L'apparition du mot «spectaculaire » n'est, de fait, pas anodine; elle accompagne une volonté d'accorder aux éléments du spectacle une fonction signifiante (et au metteur en scène un rôle d'interprète ${ }^{4}$ ), reléguant,

1. Cette question est plus particulièrement traitée dans notre étude : «La "naissance" de la mise en scène et sa théorisation », à paraître dans Roxane Martin et Marina Nordera (dir.), Les Arts de la scène à l'épreuve de l'Histoire. Les objets et les méthodes de l'historiographie des spectacles produits sur la scène française (1635-1906), Paris, Champion, 2011.

2. «Le Théâtre du Vieux-Colombier», sept. 1913, rééd. in Critiques d'un autre temps, Paris, Éd. de la Nouvelle Revue Française, 1923, p. 246.

3. Ibid., p. 248. C'est l'auteur qui souligne.

4. Notons que la rénovation dramatique souhaitée par Copeau repose précisément sur l'avènement du metteur en scène défini non plus comme simple régisseur responsable de la partie «matérielle» du spectacle, mais comme l'interpréte unique de l'œuvre dramatique. Cette distinction établie entre le 
pour s'imposer, les pratiques scéniques du théâtre «à grand spectacle » dans les contrées de la marginalité. Le spectacle qui donne sens ${ }^{1}$ (la mise en scène) vs le spectacle qui cherche le débordement des sens (le spectaculaire) est donc l'antagonisme qui caractérisait désormais - pour les théoriciens - la production théâtrale française; il révèle un changement dans la façon de concevoir le rôle social, politique et pédagogique du théâtre.

\section{ENJEUX ET MISE EN JEU DU SPECTACULAIRE : L'EXEMPLE DE LA FÉERIE}

Quand on pense à l'importance accordée au "théâtre populaire » par les acteurs de la décentralisation théâtrale - parmi lesquels on distingue de nombreux héritiers de Copeau ${ }^{2}-$, on ne peut que s'interroger sur les implications politiques qui soustendent la marginalisation du théâtre «spectaculaire ». La féerie peut aider l'historien à en repérer quelques-unes. Puisant dans le registre du merveilleux ses thèmes, ses motifs narratifs et ses personnages, elle fut sans doute la forme la plus encline à céder

régisseur et le metteur en scène est le facteur essentiel sur lequel se fondent les historiens pour célébrer la naissance de la mise en scène "moderne » qu'ils font généralement se correspondre avec l'ouverture du Théâtre-Libre d'André Antoine en 1887. Voir, par exemple, l'ouvrage récent de Bénédicte Boisson, Alice Folco, Ariane Martinez, La Mise en scène théatrale de 1800 à nos jours, Paris, PUF, 2010.

1. Comprendre par là: qui révèle le sens d'une œuvre dramatique considérée désormais comme autonome et élaborée en aval du travail de mise en scène. Toute la réforme de la mise en scène dite "moderne » repose sur une conception tout à fait nouvelle; le théâtre est désormais perçu, selon la formule d'Henri Gouhier, comme un art à deux temps : le temps de la création littéraire et celui de la création scénique. La mise en scène est donc bien une « interprétation » de l'œuvre du poète dramatique, d'où l'insistance de Copeau sur l'inopérance des effets spectaculaires pour la mise en scène des «grands ouvrages classiques». En amont de cette réforme, le texte n'est pas perçu comme indépendant du travail scénique; écriture dramatique et réalisation scénique sont interdépendantes. Ce facteur est essentiel pour comprendre les enjeux du théâtre aux XVII ${ }^{\mathrm{e}}$-XIX ${ }^{\mathrm{e}}$ siècles et analyser la manière dont le spectacle conditionne l'écriture théâtrale, donc pour l'étude du spectaculaire.

2. Sur ce point, voir l'étude de Pascale Goetschel: «Directeurs de Centres Dramatiques Nationaux: identités et différences (1945-1981)», in Pascale Goetschel et Jean-Claude Yon (dir.), Directeurs de théatre, XIX'e-XX'e siècles, Histoire d'une profession, Paris, Publications de la Sorbonne, 2008, p. 87-110. 
aux attraits du «grand spectacle ${ }^{1}$. Pourtant, la féerie ne fut pas toujours « spectaculaire » dans le sens où les éléments du spectacle ne furent pas toujours utilisés par elle pour servir une esthétique de l'effet. Un détour par son histoire, entre les XVII ${ }^{e}$ et $\mathrm{XIX}^{\mathrm{e}}$ siècles, semble donc nécessaire afin d'identifier les enjeux politiques, esthétiques et sociaux qui se cachent derrière le recours au " grand spectacle », et que la féerie a su parfois révéler par le biais de la parodie.

\section{La féerie sous l'Ancien Régime: spectaculaire et parodies}

Dans son étude sur le "conte à la scène », Christelle BahierPorte l'affirme clairement :

... le théâtre du merveilleux est sans aucun doute un théâtre de l'effet qui se fonde sur l'art de la mise en scène et des machines. C'est d'ailleurs le trait commun à toutes nos adaptations de contes, quelle que soit la scène à laquelle elles se destinent. $^{2}$

Aux $\mathrm{XVII}^{\mathrm{e}}$ et $\mathrm{XVIII}{ }^{\mathrm{e}}$ siècles, toutes les scènes semblent à même de mobiliser une machinerie théâtrale sophistiquée afin de servir un merveilleux scénique fondé sur des apparitions et des transformations à vue. Le féerique se trouve ainsi exploité aussi bien par les auteurs de la Foire, que par ceux de la ComédieItalienne, de l'Opéra, de la Comédie-Française, des théâtres de Société, des théâtres du Boulevard ${ }^{3}$. Toutefois, il convient de déterminer si le recours à la machinerie théâtrale est un facteur suffisant pour qualifier l'œuvre de «spectaculaire». Car comme le précise Christelle Bahier-Porte: «Sur les théâtres de la Foire, les auteurs rivalisent d'imagination pour montrer le merveilleux avec

1. Copeau, d'ailleurs, ne manque de bâtir le lien entre «spectaculaire » et féerie, allant jusqu'à les confondre: "nous avons la conviction profonde qu'il est désastreux pour l'art dramatique de lui ménager un grand nombre de complexités extérieures. Elles énervent, détendent sa force. Elles favorisent la facilité, le pittoresque, et font verser le drame dans la féerie » (Jacques Copeau, op. cit., p. 248-249).

2. Christelle Bahier-Porte, «Le conte à la scène, Enquête sur une rencontre (XVII ${ }^{\mathrm{e}}$ XVIII ${ }^{\mathrm{e}}$ siècles) », Féeries, 4/2007, mis en ligne le 24 oct. 2008, http:// feeries.revues.org/index223.html, p. 6.

3. Une liste chronologique des pièces illustrant le théâtre merveilleux des XVII et XVIII e siècles est donnée par Christelle Bahier-Porte dans le $\mathrm{n}^{\circ} 4$ de la revue Féeries (op. cit.). 
des moyens moindres qu'à l'opéra $»^{1}$. Un constat similaire est dressé par Nathalie Rizzoni qui, après avoir analysé les modalités du comique dans la féerie foraine des années 1710-30 - dans laquelle elle note l'utilisation de ressources scéniques "souvent rudimentaires $\gg-$, conclut :

Nos dramaturges ont exploité la veine comique propre aux situations surnaturelles des contes avec une impudente liberté ; ils l'ont fait en s'appuyant sur les propriétés de cet art vivant qu'est le théâtre - dont ils ont régulièrement repoussé les limites avec une inventive vitalité - et en faisant du spectateur un complice de la fabrique de l'illusion plutôt que le témoin ébloui de pièces « à grand spectacle ».2

L'une des caractéristiques de l'écriture féerique foraine se situe en effet dans le burlesque et le recours à la parodie. De fait, si les techniques de l'illusion sont mobilisées, elles le sont généralement pour se moquer des machines spectaculaires qui sont le propre de l'Opéra. Car le théâtre «à grand spectacle» semble avant tout l'apanage des théâtres privilégiés. Très prisé à la cour tout au long du XVII ${ }^{\mathrm{e}}$ siècle - l'on pense notamment au faste des ballets de cour, aux grandes fêtes de Versailles, aux pièces entièrement conçues autour des machines dessinées par Torelli telle l'Andromède (1650) de Pierre Corneille - le «grand spectacle» se voit circonscrit, dès la fin du $\mathrm{XVII}^{\mathrm{e}}$ siècle, à la tragédie lyrique et au ballet. Condamné par les néo-aristotéliciens, ou admis uniquement lorsqu'il satisfaisait la vraisemblance, le merveilleux - au sens de merveille, du latin mirabilia : "chose étonnante »- est alors pris en charge par les genres «modernes", c'est-à-dire par ceux qui n'existaient pas dans l'Antiquité et pouvaient donc recourir à des poétiques nouvelles, dégagées de l'emprise de l'aristotélisme. Ce fut le cas de l'opéra qui, comme le précise Guy Spielmann, « contrairement à la tragédie, montr[ait] plutôt qu'il décri[vait] », et pour lequel « la monstration ne consist[ait] pas à matérialiser ce qui [était] énoncé ou évoqué dans le discours », mais "relev[ait] d'un autre type de poétique, indépendant du premier, dont la responsabilité écho[yait] au décorateur $»^{3}$. De fait :

1. Christelle Bahier-Porte, art. cit.

2. Nathalie Rizzoni, "Féerire à la foire », Féeries, 5/2008, mis en ligne le $1^{\text {er }}$ sept. 2009, http:// feeries.revues.org/index691.htlm, p. 2 et p. 16.

3. Guy Spielmann, «Poétique(s) du merveilleux dans les arts du spectacle aux XVII - XVIII" siècles ", Georgetown University, 2007, Texte édité dans David Wetsel et Frédéric Canovas (dir.), La Spiritualité, L’Épistolaire, Le 
...les scènes à effets devinrent très rapidement des figures imposées dans la composition des œuvres lyriques, non seulement sur le plan scénographique (entrées de gloire, apparitions de démons, scènes de rêves), mais aussi dans une dimension purement musicale. ${ }^{1}$

L'opéra, genre "spectaculaire », prit donc en charge les exigences de pompe qui, selon Christian Delmas, étaient le propre des tragédies représentées à la cour au $\mathrm{XVII}^{\mathrm{e}}$ siècle $^{2}$. Autant dire qu'il satisfaisait, à la suite des pièces à machines, le goût d'une élite pour le "grand spectacle», révélateur de son prestige et de sa suprématie sociale.

Sur les théâtres de la Foire, en revanche, le recours aux machines ne semble pas uniquement orienté vers une recherche de l'effet, mis à part peut-être celui qui provoque le rire. Car, précise Christelle Bahier-Porte :

...si les scènes qui accueillent le merveilleux du conte empruntent aux prestigieuses machines de l'opéra, elles s'en moquent également comme d'un ressort obligé du merveilleux théâtral : une caisse de bois, une baguette, un jeu d'acteur, une danse peuvent alors remplacer avantageusement une machine spectaculaire. ${ }^{3}$

Cette mise en abîme du spectaculaire semble d'autant plus intéressante pour l'historien qu'elle demeure une constante de l'écriture féerique jusqu'à la deuxième moitié du XIX ${ }^{\mathrm{e}}$ siècle. Les apparitions, transformations à vue et vols qui, sur les scènes des théâtres privilégiés, sont conçus pour être impressionnants, sont dévoilés sur les petites scènes comme machines, c'est-à-dire comme simples ficelles. Jouant sur les décalages entre contenu et forme - qui est le propre de la parodie -, les vaudevillistes transforment les somptueuses gloires de l'opéra en casserole, réchaud ou marmite sur lesquels s'envole Arlequin (Les Écriteanx des fêtes parisiennes, [1710-15]), les terribles vautours en dindons (Les Petites Danaïdes, 1819), les magnifiques changements de décor de la

Merveilleux au Grand Siècle, Gunter Narr Verlag Tübingen, 2003 et consultable en ligne à l'adresse : http://opsis.georgetown.edu/articles.htm.

1. Guy Spielmann, loc. cit..

2. Voir «Introduction », in Pierre Corneille, Andromède, Christian Delmas (éd.), Paris, Didier, 1974, p. XX.

3. Christelle Bahier-Porte, loc. cit. 
grande féerie en situations cocasses (Fine-Rosée, 1857) ${ }^{1}$. Les moyens du spectaculaire, mis en œuvre par des genres qui, si l'on considère les scènes où ils étaient joués, s'adressaient à une élite sociale, sont ainsi parodiés, dénoncés comme de simples techniques visant à produire l'illusion. Autant dire que les féeries de la Foire, puis du Boulevard, engagent les moyens matériels du spectacle vers une tout autre finalité; elles ne sont pas mises au service d'une «esthétique du prodige» pour reprendre la formule de MarieFrançoise Christout $^{2}$, mais se font les vecteurs d'une métathéâtralité par l'intermédiaire de laquelle le théâtre explore sa propre capacité à créer l'illusion, et désamorce aussi peut-être, en provoquant l'éclat de rire, la recherche de sidération du théâtre spectaculaire.

Comme l'explique clairement Nathalie Rizzoni, la propension de la Foire à déjouer les conventions du théâtre «spectaculaire » doit être analysée à l'aune des conflits qui opposent régulièrement les scènes foraines aux théâtres privilégiés. D'ailleurs, ce type de féerie parodique réapparaît chaque fois que le système des privilèges est renforcé par le gouvernement en place. C'est le cas, par exemple, sous l'Empire et la Restauration où l'Opéra renoue avec la tradition de l'Ancien Régime en programmant des opérasféeries qui empruntent à la tragédie lyrique ses machines et son goût pour le merveilleux. Ces pièces sont alors immédiatement parodiées en vaudevilles-féeries sur le Boulevard. C'est le cas également pendant la période «autoritaire » du Second Empire où le "grand spectacle », qui n'était désormais plus seulement traduit par l'opéra mais aussi, entre autres, par la féerie des grands théâtres privés, favorise l'écriture de féeries parodiques sur les petits

1. Pour exemple, citons un passage de cette pièce où, pour parodier les somptueux changements de décors de la grande féerie qui faisait passer en un rien de temps les personnages du ciel à l'enfer, le héros souligne le changement de décor en s'indignant: "Alors, vous allez nous mener dans le chemin du paradis. (Sur un geste de la fée, un nuage descend, et couvre le théâtre.) Les deux extrêmes en une minute... l'eau et le feu... le chaud et le froid... je suis capable d'en avoir une fluxion de poitrine...» Sur quoi l'héroïne réplique : «Taitoubec... je te ferais de la tisane...» (Fine-Rosée, ou la Reine des Ondines, pièce féerie en 2 actes et 8 tableaux, ms., 1857, (I, 9), $\left.\mathrm{f}^{\circ} 31\right)$.

2. Voir Marie-Françoise Christout, «Techniques et effets du merveilleux dans le ballet et les arts voisins ", Revue d'histoire du théâtre, 1963-1, p. 85-93. 
théâtres du Boulevard ${ }^{1}$. Le spectaculaire semble ainsi chaque fois générer son démantèlement par le biais de la parodie. Au cœur de cette rencontre paraissent se jouer non seulement des règlements de compte entre théâtres privilégiés et théâtre indigents, mais aussi des oppositions d'ordre esthétique et - puisque les deux sont liés politique.

Cette hypothèse est corroborée par un autre type de féerie qui voit le jour dans la deuxième moitié du XVIII ${ }^{\mathrm{e}}$ siècle et qui demeure très influencée par l'esthétique du "genre sérieux » théorisé par Diderot. Sur les scènes de la Comédie-Italienne, de l'Opéra-Comique, des théâtres de Société, puis du Boulevard, s'épanouit une féerie «morale» qui délaisse les machines spectaculaires au profit d'un «merveilleux amoureux $»^{2}$. L'objectif n'est alors pas de servir une esthétique de l'effet, mais d'explorer les métamorphoses du cœur et les qualités morales qu'elles sont susceptibles d'éveiller chez le sujet amoureux. Les intrigues se concentrent sur le chemin initiatique d'un héros qui, au fur et à mesure des épreuves, accomplit son éducation morale. Ce type de pièces réalise le programme des philosophes des Lumières qui souhaitaient faire du théâtre un outil d'éducation. Quand on sait que le «troisième genre » défendu par Diderot fut aussi un moyen de dénoncer le goût d'une aristocratie pour le libertinage, la vie oisive et les fêtes fastueuses, on ne peut que constater le partage des publics qui s'opère autour du théâtre «à grand spectacle ». Sous l'Ancien Régime, celui-ci semble surtout associé au divertissement des élites ${ }^{3}$. Il en fut tout autre après la Révolution.

1. Sur ce point, nous renvoyons à notre étude : La Féerie romantique sur les scènes parisiennes 1791-1864, Paris, Champion, 2007.

2. Dans les années 1780, les théâtres du Boulevard programment des féeries de ce type de façon, visiblement, à prouver la «haute moralité » de leur répertoire et à adoucir les jugements d'une bourgeoisie qui déconsidérait leur expansion. Audinot, directeur de l'Ambigu-Comique, exprime clairement cet objectif dans la préface du Prince Noir et Blanc (féerie en 2 actes, Paris, Cailleau, 1782).

3. Marie-Emmanuelle Plagnol-Diéval montre que les théâtres de société, qui développaient un théâtre éducatif et moral, pouvaient également avoir recours au grand spectacle lorsqu'il s'agissait d'honorer un personnage illustre : «Ces fastes, liés à un certain type de sociabilité, qui renvoient au groupe une image volontairement construite de lui-même s'organisent autour d'une idée, le plus souvent empruntée aux fêtes princières ou aux manifestations organisées par les villes lors du passage d'un personnage célèbre " ("Théâtres privés et contes de fées dans la seconde moitié du XVIII" siècle ", in Féeries, 4/2007, loc. cit..). 
La loi Le Chapelier de 1791, en abolissant les privilèges dramatiques, favorisa l'élargissement des publics et encouragea la fédération de spectateurs qui jusqu'alors se répartissaient selon un clivage entre théâtres pour le peuple et pour l'élite.

\section{Après la Révolution: les enjeux du " grand spectacle »}

Si la féerie ne fut à proprement parler jamais «spectaculaire » sous l'Ancien Régime, elle le devint après la Révolution. Tout d'abord, pour une courte période après la chute du régime de Robespierre, ensuite de façon plus durable après les événements de juillet 1830. Ces événements politiques ne sont bien sûr pas étrangers au façonnement de sa nouvelle dramaturgie. Déjà parce que la Révolution avait donné le goût du spectacle à un public, désormais élargi au populaire ${ }^{1}$, qui cherchait au théâtre à voir transposées en mythes et en merveilles les situations de violence que la rue et les assemblées avaient banalisées. Le "grand spectacle » devint ainsi l'un des enjeux nouveaux de la composition dramatique. Ensuite parce que le théâtre, surtout après la Terreur, se devait de permettre à un public, en grande partie désorienté, de renouer avec les valeurs morales et religieuses. La féerie, comme le mélodrame, assuma cette fonction. Dès 1797, elle édifia une structure narrative - empruntant d'ailleurs beaucoup à la féerie morale du XVIII ${ }^{\text {e }}$ siècle - qui ne fut plus jamais remise en cause. Il s'agissait d'exploiter les amours contrariées d'un couple d'amants tourmenté par un génie maléfique, terrible tyran répandant crimes et violences parmi l'humanité. Après de nombreuses épreuves, toujours accompagnées d'effets scéniques éblouissants qui renforçaient le pathétique de la situation, les amants étaient sauvés par une fée bienfaisante qui condamnait le tyran par un châtiment exemplaire et "spectaculaire » - il pouvait par exemple être avalé par un lac de feu, dévoré par des fauves, anéanti dans un tremblement de terre. En utilisant les ressources matérielles du spectacle pour servir une finalité pédagogique et morale, la féerie de la décennie 1797-1807 cherchait à susciter l'adhésion émotive du spectateur ; car, comme le précise la fée Zatima dans L'Enfant $d u$ malheur (1797), la féerie souhaite alors montrer que derrière le masque de l'autorité souveraine se cache souvent le visage du

1. La loi Le Chapelier de 1791, en supprimant les privilèges dramatiques, avait permis le développement des théâtres privés qui pouvaient dorénavant allier le discours, la danse, la musique, etc. 
despote : «Et vous, peuple, apprenez à vous défier des apparences trompeuses; sachez que si le crime peut triompher un moment, il reçoit tôt ou tard la juste punition que le ciel lui destine... ${ }^{1}$ Autant dire qu'elle exorcisait les violences de la Terreur en promettant l'existence d'une providence sachant récompenser les hommes à leur juste valeur. Cette féerie, tout entière centrée sur l'individualisme et la rébellion face au pouvoir institutionnel, ne survécut pas aux décrets napoléoniens de 1806-07. Elle réapparut néanmoins dans les années 1830.

\section{Après 1830 : Spectaculaire et romantisme}

La féerie des années 1830-50, tout en conservant le schéma structurel de la féerie postrévolutionnaire, abandonna le pathétique. Il ne s'agissait plus de mobiliser les éléments du spectacle pour soutenir une finalité pédagogique et morale, mais de créer un théâtre de l'imaginaire pur. Le recours aux "clous» scéniques, c'est-à-dire aux effets spectaculaires, était un moyen de plonger le spectateur dans un monde imaginaire, totalement affranchi des lois de la physique et de la pesanteur. Il faut alors analyser cette féerie en la mettant en parallèle avec les drames de la période romantique. Si ces derniers cherchent, en intégrant les éléments du spectacle au sein de leur intrigue, à créer l'émotion tout en pointant les problèmes sociaux et politiques de leur époque, la féerie choisit d'explorer toujours plus loin les techniques récentes de la mise en scène afin d'emporter le spectateur loin de son quotidien. Aussi la féerie participe-t-elle pleinement du romantisme dans la mesure où elle abolit les frontières qui séparent visible et invisible, réel et irréel, et dévoile que le sens n'est pas compris dans la matière mais dans la force ('imagination) qui la traverse et révèle son caractère illusoire. En mobilisant les ressources du spectaculaire jusqu'à conduire l'espace scénique au bord de la saturation et, par ce biais, à remettre en cause les limites mêmes de la théâtralité, la féerie montre que la forme, la matière, elle-même, est illusion. C'est pourquoi elle a suscité l'enthousiasme de nombreux auteurs romantiques à cette époque ; elle satisfaisait l'autre versant du romantisme théâtral, non pas celui qui se charge d'une mission civilisatrice, mais celui qui

1. Jean Cuvelier, L'Enfant du malheur, ou les Amants muets, comédie-féerie en 4 actes, jouée sur le Théâtre de l'Ambigu-Comique à partir du 9 germinal an V (29 mars 1797), Paris, Barba, an VI, (IV, 8), p. 31. 
accorde une place d'honneur au rêve, cette percée par-delà la matière qui seule permet l'accès à l'autre monde, celui de l'imaginaire, espace de vérité pour les romantiques. C'est cette même féerie qui, dans les années 1860-1900, donna naissance à la "grande féerie » du Châtelet. Le genre toutefois, après la destruction du Boulevard du Temple, en 1862, ne s'adressait plus au même type de public; les grands théâtres privés, davantage soumis à la concurrence, engageaient des frais de mise en scène colossaux qui eurent pour conséquence une augmentation du prix des places. En outre, surenchérissant toujours sur les mêmes ficelles et ne pouvant plus explorer plus loin la fabrique de l'illusion, le genre se confronta à ses propres limites. Le cinématographe lui ouvrit de nouvelles perspectives.

Le spectaculaire, notion complexe et difficile à circonscrire, n'est donc pas à confondre avec le spectaculeux. Il ne désigne pas nécessairement le trop-plein de spectacle et n'est pas à saisir au cœur d'une profusion des images. Son essence réside avant tout dans l'effet qu'il produit sur le spectateur. C'est pourquoi Isabelle Moindrot peut écrire : "L'effet doit disparaittre avant que l'on ait $\mathrm{pu}$ expliquer les choses, effacer l'impression, retenir l'effet par le manteau ${ }^{1}$, sans quoi le spectaculaire deviendrait le spectaculeux. Car la particularité du spectaculaire est d'anéantir la frontière entre scène et salle, de dissoudre le recul critique du spectateur. C'est ce qui explique la méfiance, ou l'admiration, qu'il a pu susciter. Car l'adhésion émotive du spectateur peut parfois sembler nécessaire lorsqu'il s'agit, selon une vision rousseauiste, de transformer la foule en peuple, c'est-à-dire de cimenter les individus en un corps social par le biais de l'émotion ressentie devant le spectacle de la vertu malheureuse. C'est sur ce terreau que se sont bâties les dramaturgies du "genre sérieux», du mélodrame, de la féerie postrévolutionnaire, des drames romantiques. L'adhésion émotive $\mathrm{du}$ spectateur peut aussi être sollicitée lorsqu'il s'agit de le transporter dans le domaine de l'imaginaire, force métaphysique qui permet d'extirper pour un temps l'individu du corps social afin de lui faire vivre l'expérience du rêve, de la plongée dans l'espace intime du dedans. C'est ce que propose la féerie dans les années 1830-50. L'adhésion émotive du spectateur peut enfin sembler nécessaire lorsqu'il s'agit d'utiliser le spectaculaire comme

1. Isabelle Moindrot, loc. cit.., p. 11. 
instrument de propagande politique. Nous n'avons pas rencontré d'exemples probants illustrant ce point dans cette étude, mais il faut toutefois souligner que le spectaculaire peut aussi être un moyen pour diffuser le prestige d'un gouvernement, d'un groupe social, et affirmer sa domination culturelle. Ce fut vraisemblablement le rôle, entre autres choses, qu'ont rempli les pièces à machines, les ballets de cour, l'opéra sous l'Ancien Régime, et peut-être même la grande féerie qui, après 1862, s'adressait à un public plus fortuné, donc majoritairement bourgeois. C'est ce qui explique d'ailleurs pourquoi ces formes furent parodiées sur les petits théâtres, pourquoi aussi elles suscitèrent les diatribes des théoriciens. D’Aubignac, dans sa Pratique du théatre (1657) ne cesse de mettre en garde les auteurs sur les séductions faciles du spectaculaire, privilégiant le plaisir raisonnable, c'est-à-dire celui qui permet au spectateur de rester libre dans ses jugements et ses impressions. Copeau, puis un bon nombre des défenseurs du «théâtre d'art », condamnent également le spectaculaire, d'une part parce qu'il leur apparaît comme la caractéristique essentielle d'un théâtre bourgeois et suranné (la condamnation du spectaculaire comme indice d'un théâtre bourgeois et mercantile est clairement formulée dans les différents textes théoriques de ces metteurs en scène), d'autre part parce que le pouvoir éducatif du théâtre passe désormais par le recul critique du spectateur. De Zola à Vilar, en passant par Brecht, le théâtre se doit d'éclairer le spectateur, de l'éduquer en lui apprenant le sens critique et la faculté d'apprécier avec intelligence les beautés des grands chefs-d'œuvre de la littérature dramatique. Ainsi le spectaculaire prend-il des formes et des sens multiples selon les genres et les périodes que l'on analyse. Il demeure une question toutefois qu'il pose toujours quelles que soient les époques : celle qui interroge la nature, le pouvoir et le rôle du spectacle.

Roxane MARTIN 